\title{
DIGITAL TWINS FOR DECISION MAKING IN COMPLEX PRODUCTION AND LOGISTIC ENTERPRISES
}

\author{
WOLFGANG KUEHN \\ University of Wuppertal, Wuppertal, Germany.
}

\begin{abstract}
The operation and optimization of complex production and logistic enterprises requires modern, data and simulation driven multi criteria decision approaches. Digital enterprise systems are complex systems, in terms of layout variability, control strategies, business processes and system parameter. All of these aspects are not independent and even due to the system dynamics the optimal solution may differ depending on the actual situation applying specific requirements. In order to improve operative decision making in production and logistic enterprises a digital twin concept can be applied. These digital twins are virtual clones of real systems or subsystems. With simulation and artificial intelligence technologies new opportunities for operative decision making are possible. Digital twins can cover the entire lifecycle of an asset or process by forming a closed-loop chain for smart, connected products, services and production and logistic processes, from design to operation, from deployment to continuous improvement.
\end{abstract}

Keywords: Digital Enterprise, Digital Twin, Factory Simulation, Virtual Model

\section{INTRODUCTION}

Digitalization changes everything rapidly, it changes the daily lives as well as existing business models and creates new business opportunities. Products from all industries are getting more innovative, more data centric, and involve more software than ever. With the Internet of Things (IoT) [1] the connectivity becomes an integral component and the next generation of smart products will be more complex systems that make current development processes inadequate [2]. The industrial environment is becoming more connected. Intelligent devices and machines generate massive amounts of data, creating a bridge between real and virtual worlds [3]. Turning data into value is getting a key success factor.

\section{COMPLEX DIGITAL ENTERPRISES}

Modern digital enterprises require a holistic approach to optimize the entire value chain [4]. By use of integrated software solutions a digital image of the entire value chains can be created [5]. These digital enterprise suites have to offer an integrated portfolio of software-based systems and automation technologies for industries to seamlessly integrate and digitalize the entire value chain, including suppliers. With a Digital Twin concept, a virtual copy of the value chain can be crated [6]. This digital clone allows to simulate, to test, and to optimize in a completely virtual environment in order to reduced time to market, increased flexibility, quality and efficiency. The Digital Twin concept enables optimization in the virtual world and the subsequent transfer of results to the real world.

The operation and optimization require modern, data and simulation driven multi criteria decision approaches. Digital enterprise systems are complex systems, in terms of layout variability, complex control strategies, business processes and system parameter [8]. All of these aspects are not independent and even due to the system dynamics the optimal solution may differ depending on the actual situation and specific requirements. 


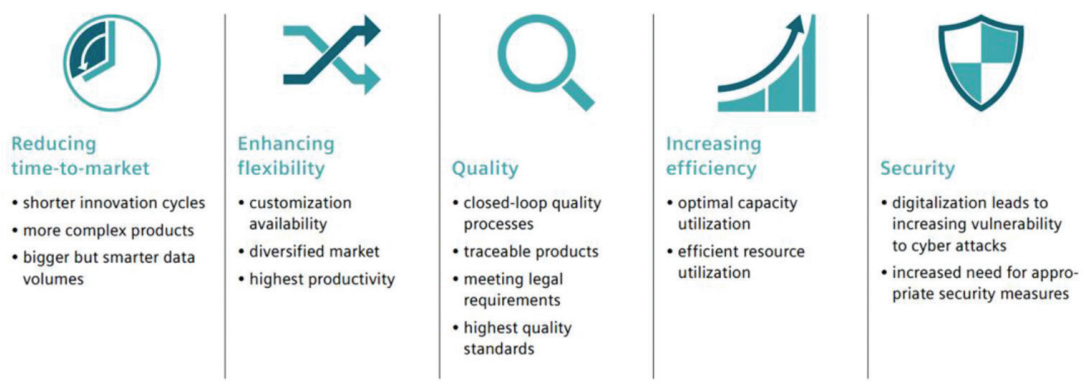

Figure 1: Enterprise Requirements (Source: Siemens 2017 [9])

\subsection{Enterprise Requirements}

In digital enterprises the engineering process has to achieve competitive advantage through improved engineering performance. Innovative engineering accelerates the design of the product and production process by enabling team collaboration to streamline the engineering processes.

In enterprises the demand for improved product performance, production flexibility and reduced operating cost are driving force of innovation, facilitated by collaborative program management across the product lifecycle and the supply chain throughout the entire product lifecycle. Within the digital enterprise the following areas are required:

- Product Lifecycle Management (PLM)

- Product Data Management (PDM)

- Manufacturing Operations Management (MOM)

- Electronic Design Automation (EDA)

- Totally Integrated Automation (TIA)

- Asset Performance Management (APM)

- Application Lifecycle Management (ALM)

Enterprises have to respond effectively to changing production schedules, which are made more challenging due to the requirements of global supply chains. The challenge of adopting new and innovative technologies and processes in order to compete successfully with increased efficiency and flexibility.

\subsection{Enterprise Decision Levels}

Complex enterprises require decisions regarding design, engineering, planning, communication and controls on different enterprise levels. Each of the particular decision requires specific algorithms and data. Therefore, standardized interfaces between these levels are mandatory.

Digital enterprises should use common data for all applications on different modelling levels in order to enable collaboration with virtual models for different purposes and different levels of detail [10]. An open architecture, open interfaces and interoperability are key factors for implementing digital enterprise concepts. 


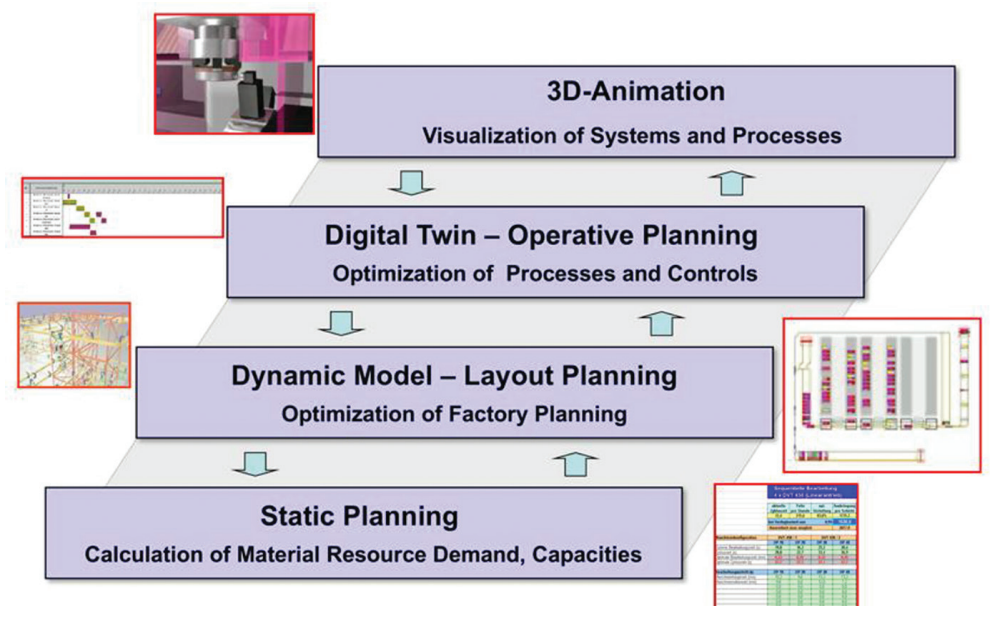

Figure 2: Enterprise decision levels

\subsection{Enterprise Optimization}

In digital enterprise systems the layout of manufacturing and logistics, the control strategies and a huge variety of parameters have to be considered and optimized [11]. Due to the complexity of digital enterprises the optimization of these systems requires multi criteria decision approaches. Most of the parameters involved are not independent and even due to the particular situation the requirements for the optimization may differ significantly. This makes optimization quite complicated, Fig. 3.

The layout-optimization has to deal with the selection and positioning of the appropriate production and logistic equipment. The case of planning new facilities is relatively seldom,

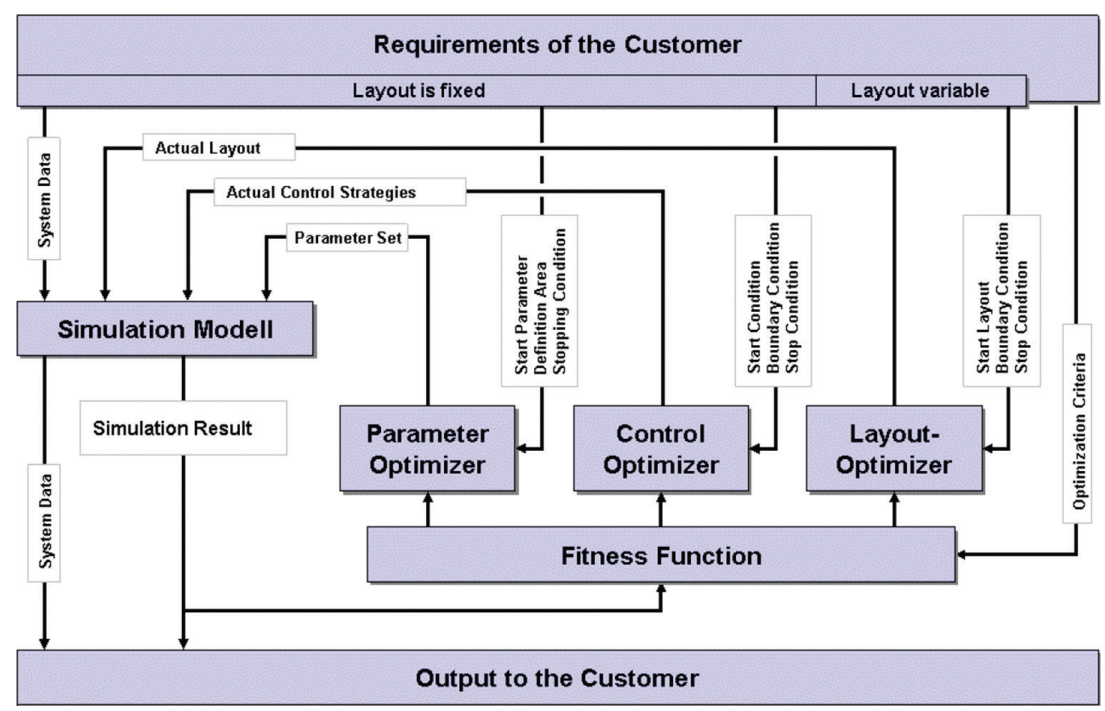

Figure 3: Optimization of parameter settings, control strategies and layout variants. 
in most cases optimization space is limited by existing resources and given boundary conditions. Major layout or structural changes in existing systems effect significant effort and investment costs.

The control optimization focuses on the improvement of the operative production and logistic control, such as scheduling and routing of orders, and technical control strategies as well. Depending on the control architecture the optimization of control strategies may require changes in the software during the ramp up phase or even during operations.

The parameter optimization is related to production parameters, such as number of work piece carriers. In most cases the implementation of parameter changes is possible with relatively small effort even during the operational phase.

All these optimization levels are interconnected and changes on one level have always an impact on the other levels. The optimization on a single level will lead to a sub-optimum. In order to improve the digital enterprise regarding factory and systems layout, control strategies and parameter settings there are complex multi criteria decision making processes required. However, optimization in the academic sense assuming that a global optimum has to be reached is not realistic in industrial practice.

The solution space for an optimization is mostly limited. A sub-optimum, which provides good or at least satisfactory results and can be reached with relatively low expense regarding time and money, may be the better solution. Due to existing boundary conditions and the relatively high investment costs for layout changes, in most industrial cases, only parameter or control changes are considered.

\section{DIGITAL TWIN CONCEPT}

The goal of Digital Twins is to monitor and improve equipment and processes in a virtual environment. Digital Twins are virtual clones of real assets or processes. As the physical source is changing, the data from the physical asset or process are collected in real-time and replicated into the virtual equivalent in order to improve operative decision making [12]. A Digital Twin can help to improve product and processes continuously.

With the technology of IoT Digital Twins can be efficiently linked to real-world objects and offer information of the state of the assets responding to changes [13]. In production and logistic enterprises Digital Twins will enable to improve operations, asset management, operational efficiency, maintenance repair and operation and insights into how products and

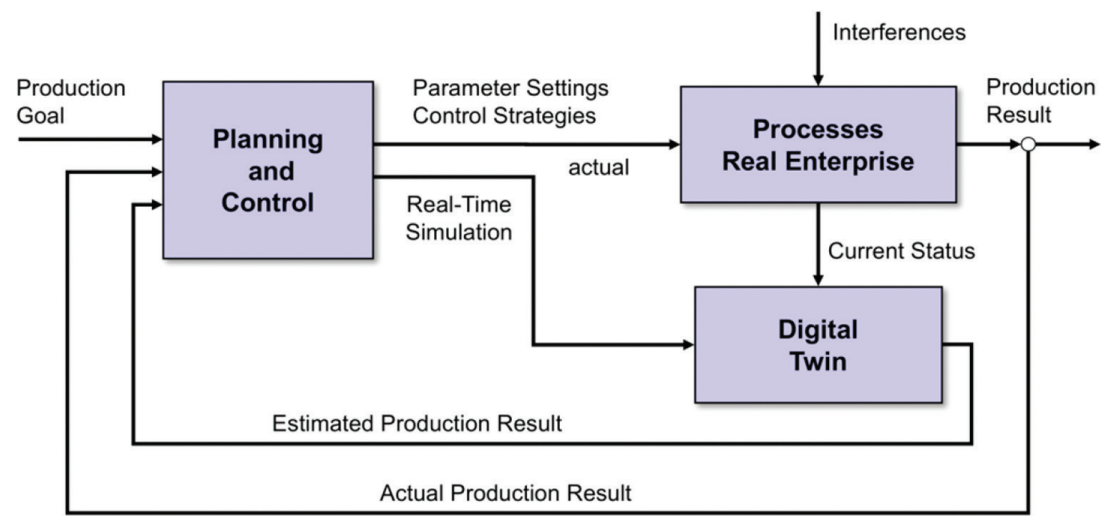

Figure 4: Digital Twin Approach 
processes can be improved [14]. The monitoring and analysis of real-time data and advanced product, production or logistic simulation models allow to improve design, controls and strategies [15]. By use of simulation models the Digital Twins can be used to operate the enterprise or parts of it in advance and to test drive several alternatives in a virtual environment before a decision is applied to the real-world system [16]. With Artificial Intelligence based capabilities Digital Twins offer for specific questions advanced product, production or logistic simulation models, which allows to test drive several alternatives in a virtual environment before decisions are applied to the real-world system [17].

\subsection{Closed Loop Approach}

The Digital Twin approach concept in Fig. 5 contains an interaction of six steps which completes a closed loop connection (physical-to-digital-to-virtual-to-physical) between the physical world and the virtual model of the Digital Twin [19].

Create - With multiple sensors various inputs from the physical process and its environment are measured by use of integrated smart components. These measurements can be classified into the operational measurements sensing physical performance criteria of the asset and the measurement of environmental or external data affecting the operations of a physical asset. Signals from the sensors may be augmented with process-based information from systems such as the manufacturing execution systems, enterprise resource planning systems, CAD models and supply chains systems in order to provide the Digital Twin with a wide range of continually updating information to be used as input for the analysis.

Communicate - Network communication enables a seamless real-time connectivity between the physical process and the digital platform. Edge processing enriches sensors and process data with business and contextual data from the environment and passes the relevant data along to the platform. Proprietary protocols are translated into standard data formats. Network technology has been enabling the Digital Twin in practice. However, with

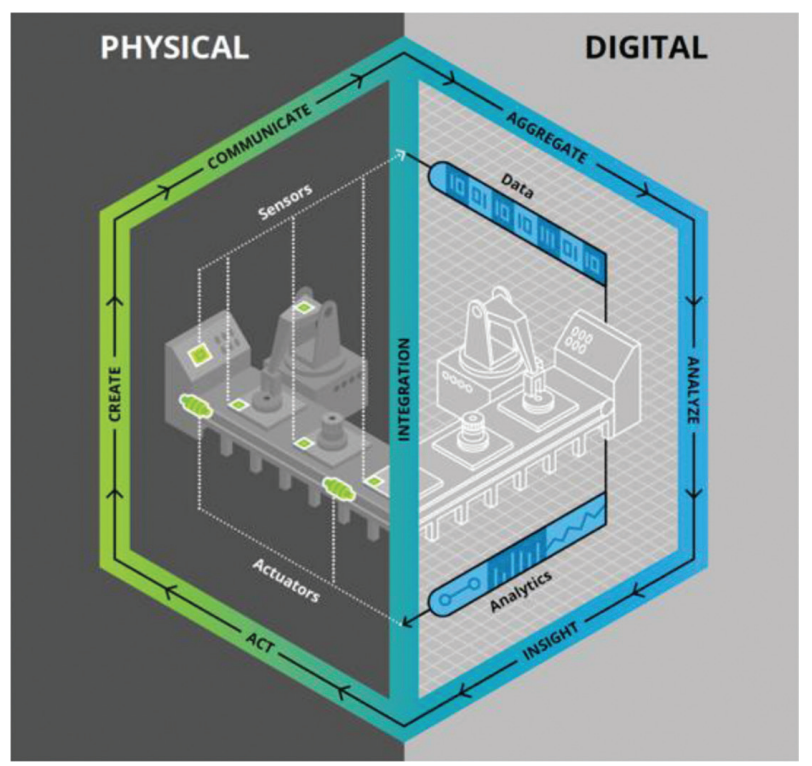

Figure 5: Manufacturing process Digital Twin model (Source: Deloitte, 2017) 
an increasing number of Iot devices security issues increase and strong security approaches are required.

Aggregate - The real-time data have to be send into a data repository, processed and prepared for the analytics. The technologies of data aggregation and processing have evolved tremendously over the last years and allow nowadays to create massively scalable architectures with greater agility.

Analyse - The aggregated data are analysed by use of advanced analytics technologies in order to analyse that data on an ongoing basis to identify opportunities for possible improvements.

Insight - Based on the analysed data, models for decision making are created. Significant differences in the performance of the Digital Twin model and the physical world indicate potentials for improvement. Simple Digital Twin models visualizes these differences in order to enable recommendations. More advanced Digital Twin models use iterative simulation to analyse with the virtual model the possible impact of changes for the real world in order to generate insights for decision making.

Act - The knowledge and recommendations from the insights step can be fed back to the physical world in order to transform the real enterprise. The insights may feed directly into actuators for movement and control, or into software systems on a higher level for operational or supply chains behaviour. With this interaction Digital Twins complete a closed loop connection from the physical world to the virtual model and back to the physical world.

These six steps are easy to list. However, it requires significant effort to achieve. Companies that embrace Digital Twins have the opportunity to better understand and continuously improvement of products, services and processes, which gives them competitive advantage [19].

\subsection{Interfacing Physical and Digital World}

For the integration in digital enterprises sensor are absolutely essential for interfacing the real word. Sensor enable to monitor and analyse multiple machine variables to improve performance, reliability, and energy efficiency of the assets. Sensors can deliver vital insights into machine state and health, e.g. indication when bearings require lubrication or are wearing out. With modern sensor technology it is possible to affordably collect huge amounts of data from hundreds of sensors in a single equipment, analyse that data in real time and detect problems before they actually happen.

Sensor technology is moving from single sensor to integrated sensor systems as devices in IoT frameworks. The performance of sensor systems has increased significantly and at the same time the cost of sensors, network hardware, computing power, data storage, and communication bandwidth have fallen dramatically. Modern integrated electronic hard- and software and standard interfaces have made powerful sensor applications cheaper and faster to implement at scale. Wireless communication and handheld or wearable devices have made access easier. Further the performance of data-analysis, even already integrated in sensor systems, has increased and is moving towards Artificial Intelligence techniques.

\subsection{Data Quality and Reliability}

Data are the key for the success of Digital Twins and any Artificial Intelligence use case. It is crucial to determine the type, quantity, and quality of data. Real-Time Data are the key factor for Digital Twins. In recent years, real-time data generated across the manufacturing value chain have grown dramatically in volume and variety. These data are coming directly from 
smart products, connected production equipment, core manufacturing processes, enterprise IT systems, and external sources from customers or suppliers.

Digital Twins require real-time data from the physical systems in order to interface between the real and the virtual world. An important issue is the quality and reliability of these data gathered from various sensors. Therefore, in industrial application Digital Twins have to apply anomaly detection of sensor data. Using a combination of physics-based knowledge and collected sensor data Digital Twins may use five types of technologies for anomaly detection available [15].

Domain or physics-based methods - Physics based models enable anomaly detection through the comparison of calculated parameters with measured values. By analyzing the expected values throughout the physical system with the reported values, it is important to determine whether a true physical anomaly is occurring or if a sensor is out of calibration. If a sensor issue is identified the physics-based models can be used to provide a virtual sensor reading based on the remaining valid data. If a true anomaly is detected, then the Digital Twin applications shall provide recommendations to minimize potential impacts.

Advanced signal processing - To detect anomalies in the presence of sensor noise advanced signal processing techniques can be used. Algorithms using wavelets, kernel regression and multi sensor data fusion techniques may be applied.

Detection by use of Statistical process control - Univariate and multivariate control chart techniques can be used with thresholds set empirically or from domain expertise. These include algorithms to detect operational regimes to apply appropriate filters and control limits.

Machine Learning anomaly detectors - Machine learning algorithms can be used, ranging from multivariate multi-level survival models to baseline asset risk, to classification techniques like logistic regression, decision trees, random forest methods, neural networks and clustering methodologies. These models are usually derived using healthy and fault data based on a database of historical sensor and configuration data.

Deep Learning Anomaly Detection - Artificial Intelligence technologies like deep learning neural networks can be trained to detect anomalies.

\subsection{Virtual Models and Automated Model Generation}

For Digital Twins it is meaningful to create lightweight virtual models by selecting required geometry, characteristics, and attributes without carrying around unnecessary details. This reduces the size of the models and allows for faster processing. Light-weight models allow to simulate complex systems, including their physical behaviours, in real-time and with acceptable costs. The time and cost of communicating is substantially less and these models can be shared within the enterprise and throughout the supplier network. This enhances collaboration, understanding and quality of product and process information.

For Digital Twins an automated model generation can be performed based on real-time data. This model generation allows a rapid and flexible modelling approach also of large production areas. The great advantage of this approach is that the model is directly generated from the current data automatically and the state of the data and the simulation model matches always. If necessary specific details may be modelled by use of predefined specific building blocks.

\subsection{Digital Thread}

A digital thread can be woven through all areas of the digital enterprise from the customer to the supplier, integrating product lifecycle management, enterprise resource planning, manufacturing operations management to customer relationship management. 


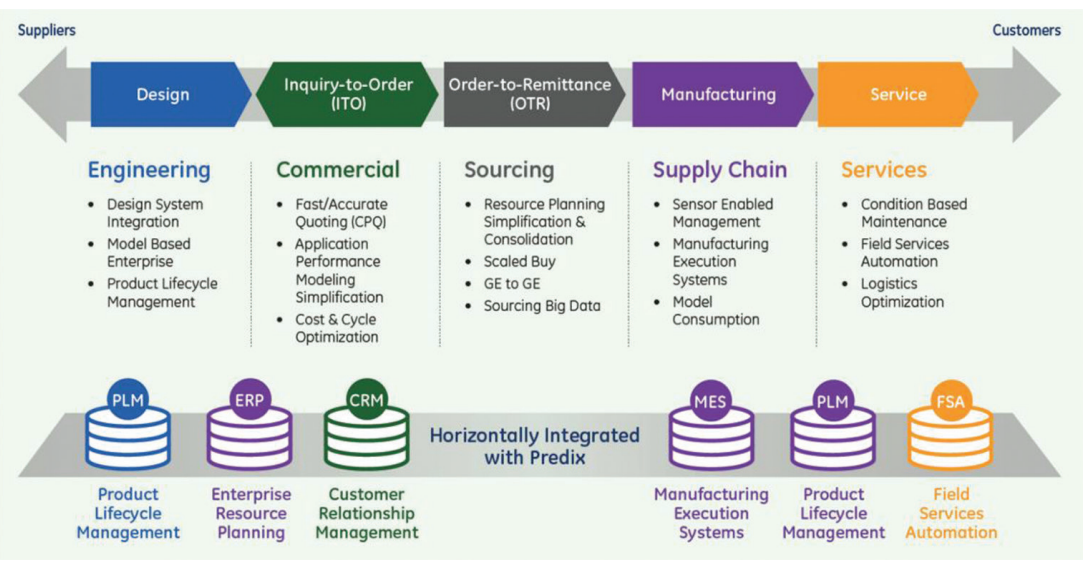

Figure 6: Digital Thread (Source: General Electric, 2016 [15])

Nowadays increasing computation power and storage possibilities of big data engines, the improvement of analytics technologies and the integration of various data enables Digital Twins to model much richer, less isolated and much more sophisticated and realistic models than ever before.

A Digital Twin conceptual architecture has to be designed for flexibility and scalability in terms of the number of sensors and messages, applied analytics and processing in order to enable the architecture to evolve rapidly with growing demands [16].

\section{ENTERPRISE DECISION MAKING}

Decision making is becoming a very important key-factor in digital enterprises. Due to the complexity of modern enterprises the operation and optimization of complex production and logistic enterprises requires data and simulation driven multi criteria decision approaches.

\subsection{Requirements}

Digital enterprise systems are complex systems, in terms of layout variability, complex control strategies, business processes and system parameter. All of these aspects are not independent and even due to the system dynamics the optimal solution may differ depending on the actual situation and specific requirements.

Decision making can be classified in decision phases and decision levels. Due to circumstances, that in digital enterprises the decision making doesn't depend on a single parameter only, multi criteria decision making is required. Complex enterprises require a high flexibility and fast, clear and save decisions.

\subsection{Rule-based Decision Making}

In complex production and logistic enterprises with a high number of different products and processes, it must be decided frequently, how jobs shall be scheduled and routed through the facilities in order to fulfil the required goals at a maximum. This planning can be generated by a rule-based scheduling strategy. A particular strategy can be built up from several priority 
classes, filtering and sorting rules, that are defined as default rules in order to optimize the global system or at least to improve local requirements. Examples for such rules are e.g. "Earliest Due Date", "Critical Ratio" (Job with the least time to due date divided by total remaining processing time) or "Critical Path".

The rules can be used as a single rule or as a network of combined rules in order to consider different aspects. Today, most companies are using enterprise software with rule-based decision making and this rule-based enterprise software has brought tremendous productivity improvements. Because enterprises are very complex and due to a relatively long reaction time and the dynamic of the systems the effects of some rules are very difficult to oversee. Therefore, in the daily business, production planners implement often only a very few of the available rules for the daily operation. Digital Twins offer a powerful approach to test various strategies and modifications without risk for the current production.

However, rule-based decision software is limited and cannot learn and improve with experience as humans can. With modern computer technology more advanced approaches will be within reach.

\subsection{Artificial Intelligence}

Decision making can be improved significantly by use of Artificial Intelligence technologies [20]. Due to advances in computer processing power, nowadays machine learning can be integrated into the decision-making processes implemented into enterprise software systems [21]. By use of advanced decision-making approaches the software will be enabled to contribute increasing levels of performance and productivity.

Advanced Digital Twins employ Artificial Intelligence technologies that leverage data from equipment to generate insights and deeper understanding of operating environments. These includes unstructured data analytics, multi-modal data analytics, component analytics, pattern recognition, learning models, knowledge networks etc. [22]. One sub-field of AI is machine learning.

\subsection{Machine Learning}

Machine Learning (ML) is an application of Artificial Intelligence in which computers perform tasks by learning from data instead of being explicitly programmed. Machine learning uses algorithms to learn from data. The more data the algorithms can access, the more the algorithms can learn. Nowadays real-world machine learning examples are everywhere, such as personalized product recommendations from Web-sites, facial recognition, or fastest route suggestions in routing applications. Machine learning is still in the early stages of industrial development and implementation, but its potential is enormous. Machine learning has the potential to discover new signals in the data that could allow for continuous improvement of complex systems.

Machine learning is based on data, either from structured data and increasingly also from unstructured data without explicitly being programmed. Based on data and algorithm it is referred to as supervised learning, where the algorithm is trained using examples where the input data and the correct answers are known and in unsupervised learning, where the algorithm must discover patterns in the data on its own, and reinforced learning, where the algorithm is rewarded or penalized for the actions it takes based on trial and error [23]. 
Digital Twins can benefit from machine learning. Very much potential is in applications that have complex rules and large amounts of data. There are some basic requirements for machine learning [24]:

Clearly formulated issue - Machine learning needs a specific, well defined task where the relevant inputs and desired output can be clearly stated. Machine learning can be valuable in discovering correlations in large amounts of data that humans could never deduced for themselves. However, it is mandatory to focus on specific tasks, it is not possible that organizations learn from all the data in the enterprise just automatically.

Meaningful differences within the dataset - Data are always the main key-factor for machine learning. Machine learning algorithms will provide useful results only, if the data to learn from contain significant meaningful differences.

Sufficient quantity of examples to learn from - Machine learning requires a lot of data. There must be enough examples to learn meaningful approximations of the decisions to be performed.

Clear definition of success - Machine learning is always evaluated by measures of performance on specific tasks. Clear evaluation criteria for the algorithm are absolutely mandatory and it is very important, that the evaluation criteria really focus on the actual problem [25].

\section{VALUE OF DIGITAL TWINS}

The Digital Twin concept is very powerful and typical benefits of this concept are:

Visibility - The Digital Twin allows visibility in the operations of an asset, an interconnected system or even a complete enterprise.

Predictive - Using various modelling techniques, Digital Twin models can be used to predict future states of an assets.

What if Analysis - Through properly designed interfaces, it is possible to interact with the model and ask what-if questions to the virtual model and to simulate various conditions that are impractical to create in real life.

Understand and explain behaviours - Digital Twin model can be used as a communication mechanism to understand and explain the behaviours of an individual asset, machine or system.

Connect disparate systems such as backend business applications - Digital Twin models can be used to connect with the backend business applications to achieve business outcomes in the context of supply chain operations including manufacturing, procurement, warehousing, transportation and logistics, field service, etc. [12].

Digital Twins offer for specific questions an advanced product, production or logistic simulation model, which allows the monitoring and analysis of real-time data to improve design, controls and strategies. Digital twins offer a possibility to operate the enterprise or parts of it in advance and to test drive several alternatives in a virtual environment before a decision is applied to the real-world system. However, the digital twin concept requires a strong interface between virtual and real world by use of real-time data. The continuous improvement is increasingly important to operate complex production and logistic enterprises on a long run efficiently and successfully. The design and realization of Digital Twins requires a powerful combination of digital plus industrial strength, a combination of deep physics knowledge, engineering design knowledge, advanced sensing and inspection technologies expertise, Artificial Intelligence and analytics experience. 


\section{REFERENCES}

[1] Menard, A., How can we recognize the real power of the Internet of Things? McKinsey, available at www.mckinsey.com/business-functions/digital-mckinsey/our-insights/ how-can-we-recognize-the-real-power-of-the-internet-of-things, 2018 (accessed 4 June 2018)

[2] Gupta, V. \& Ulrich, R., How the internet of things how the internet of things will reshape future production systems. McKinsey (Modern vision), available at www .mckinsey.com/business-functions/operations/our-insights/how-the-internet-of-thingswill-reshape-future-production-systems, 2017 (accessed 4 June 2018)

[3] Alsén, D., Patel, M. \& Shangkuan, J., The future of connectivity: Enabling the Internet of Things. McKinsey, available at www.mckinsey.com/global-themes/internet-ofthings/our-insights/the-future-of-connectivity-enabling-the-internet-of-things, 2017 (accessed 4 June 2018)

[4] Deloitte, Industry 4.0. 2017 Global Impact Report, available at www2.deloitte.com/ content/campaigns/global/global-report/global-report.html, 2017 (accessed 4 June 2018)

[5] Deloitte, Forces of change: Industry 4.0. Deloitte Development LLC, available at www2.deloitte.com/content/dam/insights/us/articles/4323_Forces-of-change/4323_ Forces-of-change_Ind4-0.pdf, 2017 (accessed 4 June 2018)

[6] Parrott, A. \& Warshaw, L., Industry 4.0 and the digital twin. Manufacturing meets its match. Deloitte, available at www2.deloitte.com/insights/us/en/focus/industry-4-0/ digital-twin-technology-smart-factory.html, 2017 (accessed 4 June 2018)

[7] Bauer, H. \& Burkacky, O., Security in the internet of things. McKinsey, available at www.mckinsey.com/industries/semiconductors/our-insights/security-in-the-internetof-things, 2018 (accessed 4 June 2018)

[8] Deloitte, The Fourth Industrial Revolution is here - are you ready? Deloitte Development $L L C$, available at www.forbes.com/forbes-insights/wp-content/uploads/2018/01/ Deloitte-FourthIndustrialRev_REPORT_FINAL-WEB.pdf, 2018 (accessed 4 June 2018)

[9] Siemens, Digital innovation. business transformation to realize innovation. Siemens, available at www.plm.automation.siemens.com/global/de/our-story/vision-digitalinnovation/, 2018 (accessed 4 June 2018)

[10] Dassault Systèmes, The digital manufacturing enterprise: Leveraging ISA-95 as a foundation. Dassault Systèmes, available at www.apriso.com/library/Whitepaper_ Digital_Manufacturing_Enterprise.php, 2017 (accessed 4 June 2018)

[11] Osan, A. \& Somers, K., Optimizing production in the age of the machine. McKinsey (Modern vision), available at www.mckinsey.com/business-functions/operations/ourinsights/optimizing-production-in-the-age-of-the-machine/, 2017 (accessed 4 June 2018)

[12] General Electric, The digital twin. Compressing time-to-value for digital industrial companies. General Electric Company, available at www.ge.com/digital/sites/ default/files/The-Digital-Twin_Compressing-Time-to-Value-for-Digital-IndustrialCompanies.pdf, 2016 (accessed 4 June 2018)

[13] Parris, C.J., Laflen, B., Grabb, M.L. \& Kalitan, D.M., The future of industrial services: the digital twin. General Electric Company, available at www.infosys.com/insights/ services-being-digital/Documents/future-industrial-digital.pdf, 2016 (accessed 4 June 2018) 
[14] Oracle, Digital twins for IoT applications. A comprehensive approach to implementing IoT Digital Twins. Oracle (White Paper), available at www.oracle.com/us/solutions/ internetofthings/digital-twins-for-iot-apps-wp-3491953.pdf, 2017 (accessed 4 June 2018)

[15] General Electric Company, GE digital twin. Analytic engine for the digital power plant. General Electric Company, available at www.ge.com/digital/sites/default/files/ Digital-Twin-for-the-digital-power-plant-.pdf, 2016 (accessed 4 June 2018)

[16] Shetty, S., How to use digital twins in your IoT strategy. Gartner, available at www .gartner.com/smarterwithgartner/how-to-use-digital-twins-in-your-iot-strategy/, 2017 (accessed 12 March 2018)

[17] Chistty, P., Prepare for the impact of digital twins. Gartner, available at www.gartner .com/smarterwithgartner/prepare-for-the-impact-of-digital-twins/, 2017 (accessed 4 June 2018)

[18] Parrott, A. \& Warshaw, L., Industry 4.0 and the digital twin. Manufacturing meets its match. Deloitte, available at www2.deloitte.com/insights/us/en/focus/industry-4-0/ digital-twin-technology-smart-factory.html, 2017 (accessed 4 June 2018)

[19] Cline, G., Product development and the centrality of digital twin. Aberdeen Group, available at www-01.ibm.com/common/ssi/cgi-bin/ssialias?htmlfid=WWL12391 USEN\&), 2017 (accessed 4 June 2018)

[20] McKinsey, An executive's guide to AI. McKinsey, available at www.mckinsey.com/ business-functions/mckinsey-analytics/our-insights/an-executives-guide-to-ai, 2017 (accessed 4 June 2018)

[21] Schick, U., What is artificial intelligence?, available at www.news.sap.com/what-isartificial-intelligence/, 2018 (accessed 4 June 2018)

[22] Gramatke, M., Gegner, J., Bauer, S., Boenisch, M. \& Le, V.D.B., Cognitive artificial intelligence the invisible invasion of the media business. Deloitte, available at www2.deloitte.com/de/de/pages/technology-media-and-telecommunications/articles/ cognitive-artificial-intelligence-in-media.html, 2017 (accessed 4 June 2018)

[23] McDermott, B, Machines can't dream. SAP, www.news.sap.com/impact-of-artificialintelligence-machines-cant-dream/, 2018 (accessed 4 June 2018)

[24] Wellers, D., Woods, J., Jendroska, D. \& Koch, C., Why machine learning and why now?, available at www.sap.com/documents/2017/05/de7cfb6d-b97c-0010-82c7eda71af511fa.html, 2017 (accessed 4 June 2018)

[25] Kolbjørnsrud, V., Amico, R. \& Thomas, R.J., How artificial intelligence will redefine management. Harward Business Review, available at www.hbr.org/2016/11/how-artificial-intelligence-will-redefine-management, (accessed 4 June 2018) 